\title{
Robust Control of Nonlinear Systems Using Model-Error Control Synthesis
}

\author{
John L. Crassidis ${ }^{1}$
}

\begin{abstract}
A new approach for the robust control of nonlinear systems is presented. This approach employs an optimal real-time nonlinear estimator to determine model error corrections to the control input using a one time-step ahead technique. Control compensation is achieved by using the estimated model error as a signal synthesis adaptive correction to the nominal control input so that maximum performance is achieved in the face of extreme model uncertainty and disturbance inputs. A significant advantage of this approach over other adaptive methods is that model parameters need not be updated online. Instead, the effects of these errors are used to update the actual control signal, which leads to a simple design strategy. The model-error control synthesis approach is used in conjunction with a variable structure controller to suppress the wing-rock motion of a slender delta wing. Simulation results indicate that the model-error control synthesis approach is extremely effective in providing closed-loop robustness in the face of significant model uncertainties and disturbance inputs.
\end{abstract}

\footnotetext{
${ }^{1}$ Assistant Professor, Texas A\&M University, Department of Aerospace Engineering, College Station, TX 77843-3141. Senior Member AIAA.
} 


\section{Introduction}

Robust control of dynamic systems is usually achieved using one of two schemes. The first scheme involves the design of a controller that is insensitive as possible to model uncertainty and/or disturbance inputs. The second scheme involves updating model parameters or control gains in real-time in order to achieve desired performance specifications. Adaptive control methods fall into this category. These control schemes can be used to provide robustness in a dynamic system with uncertainties, each with its own advantages and disadvantages.

Uncertainty in a dynamic system may take many forms, but among the most significant are noise/disturbance uncertainty and modeling/parametric uncertainty. The current design of robust controllers synthesizes a control law that maintains system responses and error signals to within pre-specified tolerances despite the effects of uncertainty on the system. Several robust control techniques have been applied to linear systems, such as $H_{\infty}{ }^{1}$ and $\mu$-synthesis ${ }^{2}$ controllers. These are based on singular value decompositions using weighted functions at varying frequencies for closed-loop specifications, which include sensitivity (disturbance), complementary sensitivity (tracking), and control input requirements. Singular values provide information in terms of guaranteed bounds on system performance or tolerable perturbations.

The control of nonlinear systems is inherently more difficult than linear systems. Variable structure (sliding mode) control $^{3}$ (VSC) is an evolving method for robust control of nonlinear systems that provides stability in the face of modeling uncertainties. Variable structure control requires that bounds on the modeling uncertainties are known in order to provide robust stability. However, "chattering" in the control signal occurs because of the conservative nature of the controller, which is highly undesirable in many systems. Chattering can be eliminated by smoothing the control signal in a thin boundary layer with a slight reduction in the controller's performance on the system.

Another class of controllers for both linear and nonlinear systems is adaptive control. These methods generally apply to systems where the dynamic structure of the model is known; but with unknown constant parameters. For linear systems, adaptive control methods have focused on the certainty-equivalence principle, ${ }^{4}$ where a particular parameter estimation technique is combined with an existing control law. The parameter estimates are treated as the true values in the control law. However, the demonstration of asymptotic behavior for nonlinear systems using the certainty-equivalence principle becomes increasingly complex. Recent approaches to this 
difficulty have focused on feedback linearization control schemes, employing a differential geometric formulation in conjunction with adaptive control. In particular, the backstepping design with tuning functions ${ }^{5}$ has been successfully shown to globally stabilize a subclass of feedback linearizable systems described in parametric strict-feedback form. A further advantage of the approach in ref. [5] is that overparametrization was removed, so that only as many controller parameters are updated as there are unknown plant parameters. This approach has been used to control a wide variety of nonlinear systems (e.g., see ref. [6]).

Disturbance attenuation is also an objective in the design of most feedback systems. Just as in the case of modeling errors, disturbance attenuation is typically achieved using one of two schemes. The first involves designing controllers that are insensitive as possible to exogenous disturbances. This is an inherent attribute in $H_{\infty}$ controllers, which has also been extended to nonlinear systems. ${ }^{7}$ Also, a worst case adaptive control-design methodology ${ }^{8}$ has recently been developed, which synthesizes a control law that meets desired asymptotic tracking and disturbance attenuation specifications. The second scheme involves canceling out disturbance effects on the output through disturbance estimation schemes. For linear systems, this has become a mainstream approach, ${ }^{9}$ where the exogenous disturbances are represented as polynomials in time with unknown coefficients that satisfy a linear differential equation. For nonlinear systems, combining variable structure control with a disturbance-accommodating observer has been developed, ${ }^{10-11}$ and has been adopted for the spacecraft attitude-control problem. ${ }^{12}$ The disturbance-accommodating observer approach has been shown to be extremely effective for disturbance attenuation; however, the performance of the observer can significantly vary for different types of exogenous disturbances, which is due to observer gain sensitivity.

The new approach developed in this paper, called model-error control synthesis (MECS), provides robust stability and performance in a controlled nonlinear system. In this approach a new control scheme is not explicitly derived. Rather, existing control techniques are combined with an optimal nonlinear estimator to determine model-error corrections which update the nominal control-input signal itself. This represents a significant departure from standard adaptive control approaches. The MECS approach has many significant advances, including: (1) system parameters need not be updated in order to achieve robust performance, (2) the largest dimension model in the MECS is equal to the plant order which leads to a computationally efficient algorithm, and (3) it can easily handle both time-varying parameter changes in the 
model and unmodeled disturbance inputs. The estimator is based upon a predictive filter for nonlinear systems. ${ }^{13}$ The main advantage of this filter is that, unlike most filter/estimation algorithms, the model error is determined during the estimation process. The predictive filter determines the corrections added to the assumed model such that the model and corrections yield an accurate representation of the actual system behavior. This new filter has been shown to be superior to the traditional Kalman filter when model error characteristics are unknown (e.g., see ref. [14-15]). If stochastic measurement errors are not present, then the filter is reduced to a state estimator only. The determined model error, which is a natural by-product of the predictive estimator, is subsequently used to update the control input in order to achieve robust performance. It will be shown that the MECS approach combined with variable structure control provides robust performance characteristics in the face of extreme parameter variations and exogenous disturbances.

The organization of this paper is as follows. First, a brief overview of the predictive filter for nonlinear systems is shown. Then, the MECS approach is summarized, followed by an analysis of the closed-loop properties. Next, a brief review of the analytical model of wing rock for slender delta wings is shown. A variable structure controller is then designed for wing-rock suppression using the nominal system parameters. Finally, results are shown using variable structure control only, and variable structure control with MECS.

\section{Predictive Filtering}

This section reviews the predictive filter (see ref. [13] for more details). In the nonlinear predictive filter it is assumed that the state and output estimates are given by a preliminary model and a to-be-determined model error vector, given by

$$
\begin{gathered}
\dot{\hat{\boldsymbol{x}}}(t)=\hat{\boldsymbol{f}}[\hat{\boldsymbol{x}}(t), t]+\hat{B}[\hat{\boldsymbol{x}}(t), t] \boldsymbol{u}(t)+\hat{G}[\hat{\boldsymbol{x}}(t), t] \hat{\boldsymbol{u}}(t) \\
\hat{\boldsymbol{y}}(t)=\hat{\boldsymbol{c}}[\hat{\boldsymbol{x}}(t), t]
\end{gathered}
$$

where $\hat{\boldsymbol{f}}: \mathfrak{R}^{n} \rightarrow \mathfrak{R}^{n}$ is the assumed model vector, $\hat{\boldsymbol{x}}(t) \in \mathfrak{R}^{n}$ is the state estimate vector, $\boldsymbol{u}(t) \in \mathfrak{R}^{q}$ is the control input, $\hat{\boldsymbol{u}}(t) \in \mathfrak{R}^{l}$ is the model error vector, $\hat{B}[\hat{\boldsymbol{x}}(t), t]: \mathfrak{R}^{n} \rightarrow \mathfrak{R}^{n \times q}$ is the assumed control input matrix, $\hat{G}[\hat{\boldsymbol{x}}(t), t]: \mathfrak{R}^{n} \rightarrow \mathfrak{R}^{n \times l}$ is the assumed model-error distribution matrix, $\hat{\boldsymbol{c}}: \mathfrak{R}^{n} \rightarrow \mathfrak{R}^{m}$ is the measurement vector, and $\hat{\boldsymbol{y}}(t) \in \mathfrak{R}^{m}$ is the estimated 
output vector. State-observable measurements are assumed for Equation (1b) in the following form

$$
\tilde{\boldsymbol{y}}(t)=\boldsymbol{c}[\boldsymbol{x}(t), t]+\boldsymbol{v}(t)
$$

where $\tilde{\boldsymbol{y}}(t) \in \mathfrak{R}^{m}$ is the measurement vector, $\boldsymbol{x}(t) \in \mathfrak{R}^{n}$ is the true state vector, $\boldsymbol{c}: \mathfrak{R}^{n} \rightarrow \mathfrak{R}^{m}$ is the true output vector, and $v(t) \in \mathfrak{R}^{m}$ is the measurement noise vector which is assumed to be a zero-mean, stationary, Gaussian white-noise distributed process with

$$
\begin{gathered}
E\{\boldsymbol{v}(t)\}=\mathbf{0} \\
E\left\{\boldsymbol{v}(t) \boldsymbol{v}^{T}(\tau)\right\}=R \delta(t-\tau)
\end{gathered}
$$

where $R \in \Re^{m \times m}$ is the covariance matrix.

A Taylor series expansion using a small $\Delta t$ of the output estimate in Equation (1b) is given by

$$
\hat{\boldsymbol{y}}(t+\Delta t) \approx \hat{\boldsymbol{y}}(t)+z[\hat{\boldsymbol{x}}(t), \Delta t]+\Lambda(\Delta t) S[\hat{\boldsymbol{x}}(t)] \hat{\boldsymbol{u}}(t)
$$

where $S[\hat{\boldsymbol{x}}(t)]: \mathfrak{R}^{n} \rightarrow \mathfrak{R}^{m \times l}$ is a generalized sensitivity matrix, and $\Lambda(\Delta t) \in \mathfrak{R}^{m \times m}$ is diagonal matrix with elements given by

$$
\lambda_{i i}=\frac{\Delta t^{p_{i}}}{p_{i} !}, \quad i=1,2, \ldots, m
$$

where $p_{i}, i=1,2, \ldots, m$, is the lowest order of the derivative of $\hat{c}_{i}[\hat{\boldsymbol{x}}(t), t]$ in which any component of $\hat{\boldsymbol{u}}(t)$ first appears due to successive differentiation and substitution for $\dot{\hat{x}}_{i}(t)$ on the right side. The $i^{\text {th }}$ component of $z(\hat{\boldsymbol{x}}, \Delta t)$ is given by

$$
z_{i}(\hat{\boldsymbol{x}}, \Delta t)=\sum_{k=1}^{p_{i}} \frac{\Delta t^{k}}{k !} L_{\hat{f}}^{k}\left(\hat{c}_{i}\right)
$$

where $L_{\hat{f}}^{k}\left(\hat{c}_{i}\right)$ is the $k^{\text {th }}$ Lie derivative, defined by 


$$
\begin{aligned}
& L_{\hat{f}}^{0}\left(\hat{c}_{i}\right)=\hat{c}_{i} \\
& L_{\hat{f}}^{k}\left(\hat{c}_{i}\right)=\frac{\partial L_{f}^{k-1}\left(\hat{c}_{i}\right)}{\partial \hat{\boldsymbol{x}}}[\hat{\boldsymbol{f}}+\hat{B} \boldsymbol{u}] \quad \text { for } k \geq 1
\end{aligned}
$$

The $i^{\text {th }}$ row of $S[\hat{\boldsymbol{x}}(t)]$ is given by

$$
s_{i}=\left\{L_{\hat{g}_{1}}\left[L_{\hat{f}}^{p_{i}-1}\left(\hat{c}_{i}\right)\right], \ldots, L_{\hat{g}_{l}}\left[L_{\hat{f}}^{p_{i}-1}\left(\hat{c}_{i}\right)\right]\right\}, \quad i=1,2, \ldots, m
$$

where $\hat{g}_{j}$ is the $j^{\text {th }}$ column of $\hat{G}(t)$, and the Lie derivative is defined by

$$
L_{\hat{g}_{j}}\left[L_{\hat{f}}^{p_{i}-1}\left(\hat{c}_{i}\right)\right] \equiv \frac{\partial L_{\hat{f}}^{p_{i}-1}\left(\hat{c}_{i}\right)}{\partial \hat{\boldsymbol{x}}} \hat{g}_{j}, \quad j=1,2, \ldots, l
$$

A loss functional consisting of the weighted sum square of the measurement-minus-estimate residuals plus the weighted sum square of the model correction term is minimized, given by

$$
\begin{gathered}
J=\frac{1}{2}[\tilde{\boldsymbol{y}}(t+\Delta t)-\hat{\boldsymbol{y}}(t+\Delta t)]^{T} R^{-1}[\tilde{\boldsymbol{y}}(t+\Delta t)-\hat{\boldsymbol{y}}(t+\Delta t)] \\
+\frac{1}{2} \hat{\boldsymbol{u}}^{T}(t) W \hat{\boldsymbol{u}}(t)
\end{gathered}
$$

where $W \in \mathfrak{R}^{l \times l}$ is weighting matrix. The necessary conditions for the minimization of Equation (10) lead to the following model error solution

$$
\begin{aligned}
& \hat{\boldsymbol{u}}(t)=-\left\{[\Lambda(\Delta t) S(\hat{\boldsymbol{x}})]^{T} R^{-1} \Lambda(\Delta t) S(\hat{\boldsymbol{x}})+W\right\}^{-1} \\
& \times[\Lambda(\Delta t) S(\hat{\boldsymbol{x}})]^{T} R^{-1}[z(\hat{\boldsymbol{x}}, \Delta t)-\tilde{\boldsymbol{y}}(t+\Delta t)+\hat{\boldsymbol{y}}(t)]
\end{aligned}
$$

Therefore, given a state estimate at time $t$, then Equation (11) is used to process the measurement at time $t+\Delta t$ to find the $\hat{\boldsymbol{u}}(t)$ to be used in $[t, t+\Delta t]$ to propagate the state estimate to time $t+\Delta t$ using Equation (1a). The weighting matrix $W$ serves to weight the relative importance between the propagated model and measured quantities. If this matrix is set to zero, then no weight is placed on minimizing the model corrections so that a memoryless estimator is given. In this case, the predictive filter reduces to a state estimator only (known as the predictive estimator, with $\tilde{\boldsymbol{y}}=\boldsymbol{y}$ ). 
Special Case

In this paper we consider the following special case for a (SISO) single-input-single-output system (which is typically used in VSC)

$$
\begin{gathered}
\hat{x}^{(n)}(t)=\hat{f}\left(\hat{x}, \dot{\hat{x}}, \ddot{\hat{x}}, \ldots, \hat{x}^{(n-1)}, t\right)+\hat{b}[u(t)+\hat{u}(t)] \\
\hat{y}(t)=\hat{x}(t)
\end{gathered}
$$

The state vector is given by

$$
\hat{\boldsymbol{x}} \equiv\left[\begin{array}{lllll}
\hat{x} & \dot{\hat{x}} & \ddot{\hat{x}} & \cdots & \hat{x}^{(n-1)}
\end{array}\right]^{T}
$$

Equation (12a) implicitly implies that the SISO system can be locally given in normal form with relative degree equal to the state dimension $(n) .{ }^{16}$ Also, any output can be used as long as the system is locally observable; however $\hat{y}(t)=\hat{x}(t)$ is chosen since this usually represents a position output in a dynamical system. The determined model error using Equation (11) with $W=0$ can be shown to be given by (dropping the time $(t)$ notation for now)

$$
\hat{u}=-\frac{n !}{\Delta t^{n}} \hat{b}^{-1}\left[\hat{\zeta}+\frac{\Delta t^{n}}{n !}(\hat{f}+\hat{b} u)-\left(y^{\Delta}-\hat{x}\right)\right]
$$

where $y^{\Delta} \equiv y(t+\Delta t)$, and $\hat{\zeta}$ is given by

$$
\hat{\zeta}=\sum_{i=1}^{n-1} \frac{\Delta t^{i}}{i !} \hat{x}^{(i)}
$$

Substituting Equation (14) into Equation (12a) yields

$$
\hat{x}^{(n)}=-\frac{n !}{\Delta t^{n}}(\hat{\zeta}+\hat{x})+\frac{n !}{\Delta t^{n}} y^{\Delta}
$$

This corresponds to a feedback linearization of the assumed dynamics.

\section{Model-Error Control Synthesis}

\section{Control Design}

The concept of model-error control synthesis is a simple one. The actual system is represented by the following 


$$
\begin{gathered}
x^{(n)}=f\left(x, \dot{x}, \ddot{x}, \ldots, x^{(n-1)}, t\right)+b u+e d \\
y=x
\end{gathered}
$$

where $d \in \mathfrak{R}^{1}$ is an exogenous disturbance and $e$ is a scalar. The control design is given by using the assumed model for the system, given by

$$
\begin{gathered}
\hat{x}^{(n)}=\hat{f}\left(\hat{x}, \dot{\hat{x}}, \ddot{\hat{x}}, \ldots, \hat{x}^{(n-1)}, t\right)+\hat{b} \bar{u} \\
\hat{y}=\hat{x}
\end{gathered}
$$

Equation (18) inherently uses the certainty-equivalence principle ${ }^{4}$ since estimated states are used. A VSC design can be used for the nominal controller, given by

$$
\bar{u}=-\hat{b}^{-1}\left[\hat{f}+\dot{s}-\hat{x}^{(n)}+\eta \operatorname{sat}\left(\frac{s}{\rho}\right)\right]
$$

where $\eta$ is a control gain and $\rho$ is the control boundary layer thickness. The sliding line $(s)$ is given by

$$
s=\left(\frac{d}{d t}+\vartheta\right)^{n-1}(\hat{x}-r)
$$

where $\vartheta$ is another control gain, $r$ represents a reference input, and the function sat is the saturation function defined by

$$
\begin{aligned}
& \operatorname{sat}(y)=y \quad \text { if }|y| \leq 1 \\
& \operatorname{sat}(y)=\operatorname{sgn}(y) \quad \text { otherwise }
\end{aligned}
$$

In the MECS approach Equation (12a) is used to compensate the control input for any general model error. Adding the model-error into the control signal is a valid since we are free to choose where to place the model-error correction in the system (the system is implicitly implied to be both controllable and observable). A block diagram of a controlled system with MECS is shown in Figure 1. First, a controller is designed using nominal system parameters, with the control signal represented by $\bar{u}$. The predictive estimator is used for both state estimation and model error determination using Equation (11). Since Equation (12a) is used to determine this model 
error, $\hat{u}$ now represents a correction to the control. In order to compensate the effects of the model errors and disturbances on the control signal, the actual control input is now given by

$$
u(t)=\bar{u}(t)-\hat{u}(t-\Delta t)
$$

with $\hat{u}\left(t_{0}\right)=0$. The model-error is taken at time $t-\Delta t$ since a response from the plant must be given before the model error can be determined. The ramifications of this time delay in the control law will be shown later. The main advantage of this update law is that the system parameters need not be updated since only their effects on the nominal system are used to update the control input, which can provide closed-loop robustness with respect to the model errors. The same concept holds true for the effects of disturbances on the closed-loop system.

Analysis

For the analysis we first make the standard assumption that the true state variables are replaced with the estimated state variables. It is well know that when no model errors and disturbances exist, the variable structure controller performs a feedback linearization. This can easily be shown by substituting Equation (22) into Equation (17a) with $\hat{u}=0, d=0$, and with $\hat{f}=f, \hat{b}=b$ (i.e., no model errors), yielding

$$
x^{(n)}=-g
$$

where

$$
g=\dot{s}-x^{(n)}+\eta \operatorname{sat}\left(\frac{s}{\rho}\right)
$$

The stability using this control law can be shown by considering the following candidate Lyapunov equation

$$
V=\frac{1}{2} s^{2}
$$

Taking the time derivative of Equation (25) yields

$$
\dot{V}=-s \eta \operatorname{sat}\left(\frac{s}{\rho}\right)
$$


which is always negative definite for positive values of $\eta$. Therefore, closed-loop stability is proven. However, if model errors and external disturbances are present, then the dynamics become

$$
x^{(n)}=\left(f-\hat{b}^{-1} b \hat{f}\right)-\hat{b}^{-1} b g+e d
$$

Equation (27) shows that parameter errors and external disturbances can clearly affect closedloop performance using VSC.

In order to demonstrate the stability of the MECS approach, we first re-write Equation (4) with the output given in Equation (17b), so that

$$
y^{\Delta}=y+\zeta+\frac{\Delta t^{n}}{n !}(f+b u+e d)
$$

with

$$
\zeta=\sum_{i=1}^{n-1} \frac{\Delta t^{i}}{i !} x^{(i)}
$$

Substituting Equation (29) into Equation (14) yields

$$
\hat{u}=\hat{b}^{-1}[(f-\hat{f})+(b-\hat{b}) u+e d]
$$

Equation (30) clearly show how $\hat{u}$ determines the model error and external disturbance. Therefore, as $f \rightarrow \hat{f}$ and as $b \rightarrow \hat{b}$, with no disturbance the determined model-error approaches zero. Note, the derivation leading to Equation (30) again uses the certainty-equivalence principle, since $x^{(i)}$ is assumed to be equivalent to $\hat{x}^{(i)}$. This is a valid assumption as long as $\Delta t$ for the Taylor series expansion in Equation (4) is small enough so that $\hat{y}(t)$ accurately approximates $y(t)$.

If the control input is assumed to be given by $u(t)=\bar{u}(t)-\hat{u}(t)$, then the following expression for the control input is given

$$
u=-b^{-1}(f+g+e d)
$$

Then the closed-loop equation for $x^{(n)}$ becomes 


$$
x^{(n)}=-g
$$

which is again the feedback linearization case with disturbance removed. Therefore, the effects of the parameter uncertainties and external disturbances have been exactly cancelled through the determined model-error in Equation (30).

For practical control purposes, Equation (22) must be used for the control law. To see the effects of this control law, we first re-write Equation (30) with a one time-step delay

$$
\hat{u}(t-\Delta t)=\hat{b}^{-1}\{[f(t-\Delta t)-\hat{f}(t-\Delta t)]+(b-\hat{b}) u(t-\Delta t)+e d(t-\Delta t)\}
$$

Next, using a one time-step delay in Equations (19) and (28), the following expressions can be written

$$
\begin{gathered}
\hat{f}(t-\Delta t)=-g(t-\Delta t)-\hat{b} \bar{u}(t-\Delta t) \\
f(t-\Delta t)=\frac{n !}{\Delta t^{n}}[y(t)-y(t-\Delta t)]-\frac{n !}{\Delta t^{n}} \zeta(t-\Delta t)-b u(t-\Delta t)-e d(t-\Delta t)
\end{gathered}
$$

Substituting Equation (34) into (33), and using the one-time delay in Equation (22) yields

$$
\hat{u}(t-\Delta t)=\hat{u}(t-2 \Delta t)+\hat{b}^{-1}\left[\frac{n !}{\Delta t^{n}}[y(t)-y(t-\Delta t)]-\frac{n !}{\Delta t^{n}} \zeta(t-\Delta t)+g(t-\Delta t)\right]
$$

Next, substituting Equation (34b) for $\zeta(t-\Delta t)$ into (35), and taking a one time-step ahead leads to

$$
\hat{u}(t-\Delta t)=\hat{u}(t)-\hat{b}^{-1}[f(t)+b u(t)+e d(t)+g(t)]
$$

Therefore, the control law becomes

$$
u(t)=-b^{-1}[f(t)+e d(t)+g(t)]+b^{-1} \hat{b}[\hat{u}(t)-\hat{u}(t-\Delta t)]
$$

A feedback linearization still occurs in the closed-loop system, but with an extra term given by $\hat{b}[\hat{u}(t)-\hat{u}(t-\Delta t)]$. From Equations (30) and (33), the error produced by this control law are given by the difference from time $(t-\Delta t)$ to $t$ of the model errors and disturbance input. Therefore, the errors caused by the extra term are in fact second-order, as opposed to the first- 
order errors affecting the standard VSC, shown by Equation (27). This leads to a more robust control design than using VSC only.

\section{Wing Rock Suppression}

In this section the MECS approach is used to suppress wing rock motion in slender delta wings. Wing rock represents a self-induced limit cycle oscillation in the presence of some initial disturbance or asymmetry in the flow field. The primary causes of wing rock are the interactions between the forebody and the wing vortices at high angles of attack. ${ }^{17}$ This has been verified using a video-based experimental investigation for wing rock of sharp-edged delta wings with leading-edge sweep angles of $70^{\circ}$ and $80^{\circ}$, which also suggests that the limit cycle behavior is due to the relative phasing between model oscillations and vortex movements. ${ }^{18}$ Analytical models of this highly nonlinear phenomenon have been developed and used to predict roll divergence as well as other characteristics in the oscillations, such as the period and amplitude of the oscillations. ${ }^{19}$

Recently, the control of wing rock has gained much attention, including, optimal control methods, ${ }^{20}$ adaptive control using backstepping, ${ }^{6}$ and variable structure adaptive control. ${ }^{21}$ One of the difficult aspects in the control of wing rock motion is the extreme sensitivity to parameter variations (i.e., a slight change in a model parameter can significantly affect overall system response). This was evident in the optimal control method in ref. [20], in which the aerodynamics parameters are assumed to be known. The backstepping design with tuning functions in ref. [6] has been successfully shown to globally suppress wing rock motion in the face of significant parametric uncertainties. A further advantage of this approach is that overparametrization was removed, so that only as many controller parameters are updated as there are unknown plant parameters. However, disturbance inputs were not considered, which can adversely affect closed-loop performance. The variable structure adaptive control approach in ref. [21] has been shown to provide good disturbance rejection capability, and is insensitive to plant nonlinearities or parametric variations. However, bounds on the uncertain functions are needed in the control design. Also, higher-order filters are required for the controller synthesis. For the interested reader, refs. [6], [20], and [21] also summarize other past control approaches for wing rock suppression.

Model-Error Control Synthesis Design

The wing-rock equation of motion for slender delta wings is given by ${ }^{19}$ 


$$
\begin{gathered}
\dot{\phi}=p \\
\dot{p}=c_{1}+c_{2} \phi+c_{3} p+c_{4}|\phi| p+c_{5}|p| p+c_{6} u+c_{6} d
\end{gathered}
$$

where $\phi$ is the roll angle, $p$ is the roll rate, $u$ represents the control surface angle, and $d$ is a control disturbance. For an angle of attack of 30 degrees the following coefficients are given ${ }^{6}$

$$
\hat{c}_{1}=5, \quad \hat{c}_{2}=-26.7, \quad \hat{c}_{3}=0.765, \quad \hat{c}_{4}=-2.9, \quad \hat{c}_{5}=-2.5, \quad \hat{c}_{6}=0.75
$$

where the symbol $\wedge^{\wedge}$ denotes nominal values. Measurements of roll only are assumed, so that

$$
y=\phi
$$

The lowest order time-derivative of Equation (40) in which the model error first appears is two. Therefore, the determined model error is given by

$$
\hat{u}=-\alpha\left[\hat{p}+\frac{\Delta t}{2}\left(\hat{c}_{1}+\hat{c}_{2} \hat{\phi}+\hat{c}_{3} \hat{p}+\hat{c}_{4}|\hat{\phi}| \hat{p}+\hat{c}_{5}|\hat{p}| \hat{p}+\hat{c}_{6} u\right)-\frac{1}{\Delta t}\left(\phi^{\Delta}-\hat{\phi}\right)\right]
$$

where

$$
\alpha=\frac{2 \hat{c}_{6} \Delta t^{3}}{\hat{c}_{6}^{2} \Delta t^{4}+4 R W}
$$

The case where $W=0$ corresponds to the feedback linearization case, yielding

$$
\left[\begin{array}{c}
\dot{\hat{\phi}} \\
\dot{\hat{p}}
\end{array}\right]=\left[\begin{array}{cc}
0 & 1 \\
-2 / \Delta t^{2} & -2 / \Delta t
\end{array}\right]\left[\begin{array}{l}
\hat{\phi} \\
\hat{p}
\end{array}\right]+\left[\begin{array}{c}
0 \\
2 / \Delta t^{2}
\end{array}\right] \phi^{\Delta}
$$

The eigenvalues of the state estimator matrix are given by

$$
s_{1,2}=\frac{1}{\Delta t}(-1 \pm j)
$$

Therefore, the estimator's dynamics are dependent only on the interval $\Delta t$. This provides a very computationally efficient routine, since a nonlinear model does not have to be integrated using numerical techniques.

The design goal is to suppress any wing-rock motion within 2 seconds. A variable structure controller has been designed to meet this requirement, given by 


$$
\begin{aligned}
\bar{u}= & -\frac{1}{\hat{c}_{6}}\left(\hat{c}_{1}+\hat{c}_{2} \hat{\phi}+\hat{c}_{3} \hat{p}+\hat{c}_{4}|\hat{\phi}| \hat{p}+\hat{c}_{5}|\hat{p}| \hat{p}\right) \\
& +\frac{1}{\hat{c}_{6}}\left\{\ddot{r}-\vartheta(\hat{p}-\dot{r})-\eta \operatorname{sat}\left[\frac{(\hat{p}-\dot{r})+\vartheta(\hat{\phi}-r)}{\rho}\right]\right\}
\end{aligned}
$$

To meet the design goal (with $r=0$ ), the following gains are used: $\eta=3, \rho=0.1$, and $\vartheta=10$.

\section{Simulation Results}

For the first simulation, the variable structure controller has been designed to bring an initial roll angle of 30 degrees to zero within 2 seconds (with $r=0$ ). For all simulations, Equations (38) and (43) have been integrated using $\phi\left(t_{0}\right)=\hat{\phi}\left(t_{0}\right)=30^{\circ}$ and $p\left(t_{0}\right)=\hat{p}\left(t_{0}\right)=0 \mathrm{deg} / \mathrm{sec}$. A plot of the response and control input with no model errors is shown in Figure 2. Clearly, the variable structure controller is able to meet the desired specification. The next case assumes an error in $c_{1}$, with $c_{1}=6$. The nominal values for the controller are still given by Equation (39). This essentially adds a constant control disturbance. A plot of the variable structure controller results without MECS $(u=\bar{u})$ is shown in Figure 3. Clearly, for this case the variable structure controller does not meet the requirement. A plot of the determined model error is shown in Figure 4. The predictive estimator is able to correctly determine the actual model error. A plot of the variable structure controller results with MECS is shown in Figure 5. Clearly, MECS provides robust performance for this case.

The next two cases show how MECS can be effective even for radical parameter changes. For the first case, the true parameters are given by $c_{1}=5 \sin (15 t), \quad c_{4}=3 \cos (5 t)$, $c_{5}=10 \sin (10 t)$. The parameter changes do not have any physical meaning; however they show how well the MECS approach can handle even time-varying parameter changes. The nominal values for the controller are given by Equation (39). A plot of the variable structure controller results without MECS is shown in Figure 6. The variable structure controller cannot remain on the sliding line since the model parameters for $c_{1}, c_{4}$, and $c_{5}$ are assumed to be constant. A plot of the determined model error is shown in Figure 7. As stated previously, the changes in the individual model parameters are not important. The MECS approach updates the control signal from the overall effects of these parameter errors. A plot of the variable structure controller results with MECS for this case is shown in Figure 8. Again, MECS provides robust 
performance. The second case involves a radical change in the model itself. Suppose that the actual system is given by

$$
\begin{gathered}
\dot{\phi}=p \\
\dot{p}=c_{1}+c_{2} \phi^{3}+c_{3} p+c_{4}|\phi| p+c_{5}|p| p+c_{6} u+c_{6} d
\end{gathered}
$$

For this system a cubic dependence in $\phi$ is present for the $c_{2}$ term. The assumed model for the variable structure controller and predictive estimator is still given by Equation (38), with nominal parameter values equal to the true parameter values. A plot of the variable structure controller results without MECS is shown in Figure 9. This again shows that variable structure control does not provide robust performance. A plot of the determined model error is shown in Figure 10, and a plot of the variable structure controller with MECS is shown in Figure 11. Again, the MECS approach provides robust performance. These two cases clearly show advantages over parameter-adaptive techniques, which typically assume that the model parameters are constant (or slowly time-varying) and that the structure of the model is well known.

In order to further test the MECS approach, a number of additional simulations have been performed. The first case involves multiplying the nominal model parameters by some constant factor $(\beta)$ to obtain the true parameters, with

$$
c_{i}=\beta \hat{c}_{i}, \quad i=1, \ldots 5
$$

Various constant factors have been used in the simulations. Table 1 gives the norm-error results in roll angle (with the trajectory in the top plot of Figure 2 as the reference) produced using both VSC-only and VSC with MECS for the 3 second simulations. Next, a change in the input value $c_{6}$ was considered only with $c_{6}=\beta \hat{c}_{6}$. Results for this case are summarized in Table 2. From this analysis, both controllers are more sensitive to variations in $c_{6}$. However, the MECS approach is able to provide robust performance when compared to VSC-only, even for extreme variations in model parameters. 
Table 1 Norm-Error Results in Roll for Model Parameter Variations

\begin{tabular}{|c|c|c|}
\hline$\beta$ & VSC & MECS \\
\hline 0.1 & 4300.00 & 0.0213 \\
0.5 & 13.2576 & 0.0118 \\
0.8 & 1.3881 & 0.0047 \\
1.1 & 0.4031 & 0.0024 \\
1.5 & 2.4160 & 0.0118 \\
2 & 4.6543 & 0.0236 \\
5 & 6.8370 & 0.0935 \\
10 & 7.2386 & 0.2075 \\
\hline
\end{tabular}

Table 2 Norm-Error Results in Roll for Control-Input Parameter Variations

\begin{tabular}{|c|c|c|}
\hline$\beta$ & VSC & MECS \\
\hline 0.1 & 7.4658 & 0.1989 \\
0.5 & 4.5660 & 0.0224 \\
0.8 & 0.8193 & 0.0056 \\
1.1 & 0.4248 & 0.0020 \\
1.5 & 2.9329 & 0.0075 \\
2 & 13.1282 & 0.2933 \\
5 & $\infty$ & 208.42 \\
10 & $\infty$ & $\infty$ \\
\hline
\end{tabular}

\section{Conclusions}

In this paper, a new approach was presented for the control of nonlinear systems with parametric and structural model uncertainties as well as external disturbance inputs. The new control approach uses an optimal nonlinear estimator to update the control signal in order to account for the effects of model errors on the system. The advantages of this approach include: (1) the determined model is a natural by-product of the state estimator, (2) no model updating is required, and (3) it easily handles time-varying system parameters. The new approach was used in conjunction with variable structure control to suppress the wing rock motion of a slender delta wing. Results indicated that the new approach is able to provide robust performance even for the case of radically varying system parameters and exogenous disturbances.

\section{Acknowledgement}

The author wishes to acknowledge F. Landis Markley from NASA-Goddard Space Flight Center, and John L. Junkins from Texas A\&M University for many helpful suggestions and 
comments. Also, this author wishes to thank Donald T. Ward from Texas A\&M University for providing documents on the experimental investigation of wing rock.

\section{References}

[1] Maciejowski, J.M., Multivariable Feedback Design, Addison Wesley Publishing Co., Wokingham, England, 1989, Chapter 6.

[2] Buschek, H., and Calise, A.J., " $\mu$ Controllers: Mixed and Fixed," Journal of Guidance, Control and Dynamics, Vol. 20, No. 1, Jan.-Feb. 1997, pp. 34-41.

[3] Slotine, J.-J.E., and Li., W., Applied Nonlinear Control, Prentice Hall, Englewood Cliffs, NJ, 1991, Chapter 7.

[4] Goodwin, G.C., and Sin, K.S., Adaptive Filtering, Prediction and Control, Prentice Hall, Englewood Cliffs, NJ, 1984, Chapter 6.

[5] Krstic, M., Kanellakopoulos, I., and Kokotovic, P.V., "Adaptive Nonlinear Control Without Overparametrization," Systems and Control Letter, Vol. 19, No. 3, 1992, pp. 177-185.

[6] Monahemi, M.M., and Krstic, M., "Control of Wing Rock Motion Using Adaptive Feedback Linearization," Journal of Guidance, Control and Dynamics, Vol. 19, No. 4, July-Aug. 1996, pp. 905-912.

[7] Isidori, A., and Astolfi, A., "Disturbance Attenuation and $H_{\infty}$-Control via Measurement Feedback in Nonlinear Systems," IEEE Transactions on Automatic Control, Vol. 37, No. 9, Sept. 1992, pp. 1283-1293.

[8] Pan, Z., and Basar, T., "Adaptive Controller Design for Tracking and Disturbance Attenuation in Parametric Strict-Feedback Nonlinear Systems," IEEE Transactions on Automatic Control, Vol. 43, No. 8, Aug. 1998, pp. 1066-1083.

[9] Franklin, G.F., Powell, J.D., and Emami-Naeini, Feedback Control of Dynamic Systems (3 ${ }^{\text {rd }}$ Edition), Addison-Wesley, Reading, MA, 1994, Chapter 7.

[10] Kim, J., "Disturbance Accommodating Sliding Mode Control," Proceedings of the American Control Conference, (Chicago, IL), American Automatic Control Council, Green Valley, AZ, Vol. 1, June 1992, pp. 888-890. 
[11] Chen, X., and Fukuda, T., "VSS Theory-Based Disturbance Estimation Scheme for MIMO Systems and Its Applications," International Journal of Control, Vol. 69, No. 6, 1998, pp. 733-752.

[12] Kim, J., Kim, J., and Crassidis, J.L., "Disturbance Accommodating Sliding Mode Controller for Spacecraft Attitude Maneuvers," Proceedings of the AAS/GSFC $13^{\text {th }}$ International Symposium on Space Flight Dynamics, NASA-Goddard Space Flight Center, (Greenbelt, MD), NASA/CP1998-206858, May 1998, pp. 119-131; see also American Astronautical Society, AAS Paper 98-310.

[13] Crassidis, J.L., and Markley, F.L., "Predictive Filtering for Nonlinear Systems," Journal of Guidance, Control, and Dynamics, Vol. 20, No. 3, May-June 1997, pp. 566-572.

[14] Crassidis, J.L., and Markley, F.L., "Predictive Filtering for Attitude Estimation Without Rate Sensors," AIAA Journal of Guidance, Control, and Dynamics, Vol. 20, No. 3, MayJune 1997, pp. 522-527.

[15] Crassidis, J.L., Lightsey, E.G., and Markley, F.L., "Efficient and Optimal Attitude Determination Using Recursive Global Positioning System Signal Operations," Proceedings of the AIAA Guidance, Navigation, and Control Conference, (Boston, MA), AIAA, Reston, VA, Aug. 1998, Part 3, AIAA Paper \#98-4496, pp. 1813-1823.

[16] Isidori, A., Nonlinear Control Systems ( $3^{\text {rd }}$ Edition), Springer-Verlag, Berlin, Germany, 1995, Chapter 4.

[17] Suárez, C.J., Kramer, B.R., Ayers, B., and Malcolm, G.N., "Forebody Vortex Control for Suppressing Wing Rock on a Highly-Swept Wing Configuration," Proceedings of the $10^{\text {th }}$ Applied Aerodynamics Conference, (Palo Alto, CA), AIAA, Reston, VA, June 1992, AIAA Paper 92-2710.

[18] Morris, S.L., “A Video-Based Experimental Investigation of Wing Rock,” Ph.D. Thesis, Department of Aerospace Engineering, Texas A\&M University, College Station, TX, Aug. 1989.

[19] Nayfeh, A.H., Elzebda, J.M., and Mook, D.T., "Analytical Study of the Subsonic Wing Rock Phenomenon for Slender Delta Wings," Journal of Aircraft, Vol. 26, No.9, 1989, pp. 805-809. 
[20] Luo, J., and Lan, C.E., "Control of Wing Rock Motion of Slender Delta Wings," Journal of Guidance, Control and Dynamics, Vol. 16, No. 2, March-April 1993, pp. 225-231.

[21] Araujo, A.D., and Singh, S.N., "Variable Structure Adaptive Control of Wing-Rock Motion of Slender Delta Wings," Journal of Guidance, Control and Dynamics, Vol. 21, No. 2, March-April 1998, pp. 251-256. 
Fig. 1 Model-Error Control Synthesis

Fig. 2 Nominal Response

Fig. 3 Variable Structure Control Without Model-Error Control Synthesis

Fig. 4 Determined Model Error

Fig. 5 Variable Structure Control With Model-Error Control Synthesis

Fig. 6 Variable Structure Control Without Model-Error Control Synthesis

Fig. 7 Determined Model Error

Fig. 8 Variable Structure Control With Model-Error Control Synthesis

Fig. 9 Variable Structure Control Without Model-Error Control Synthesis

Fig. 10 Determined Model Error

Fig. 11 Variable Structure Control With Model-Error Control Synthesis 


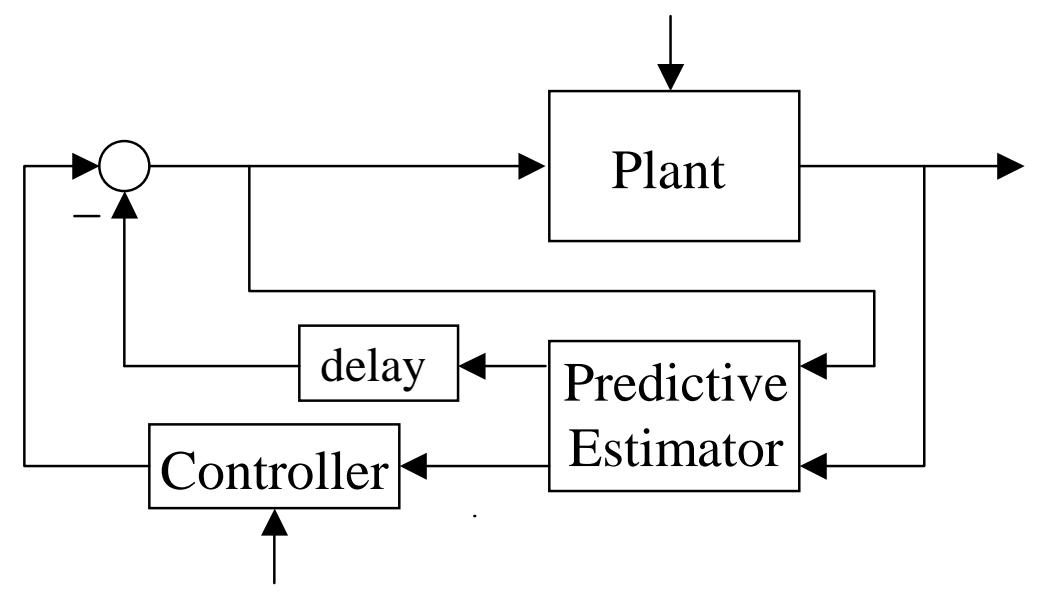



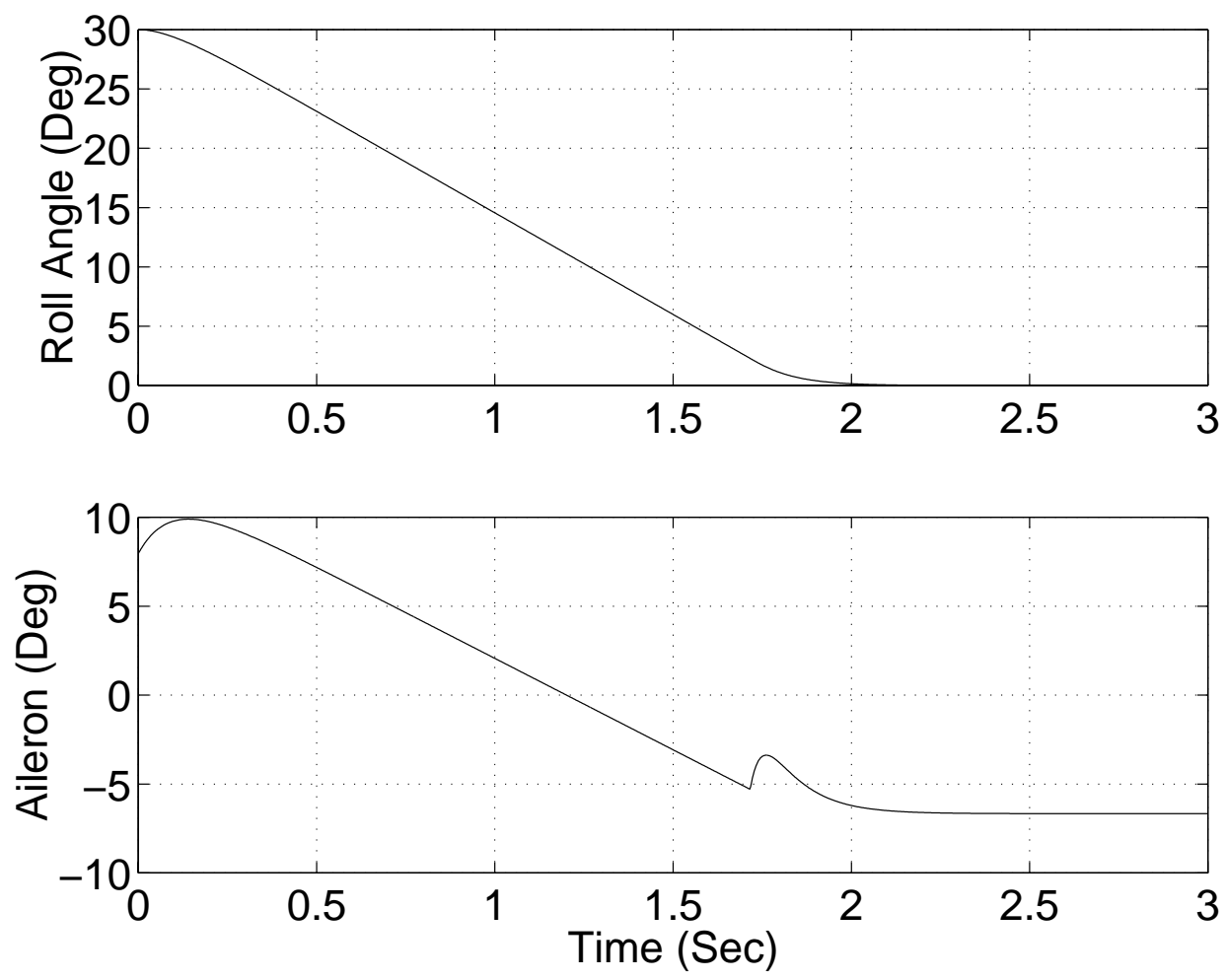

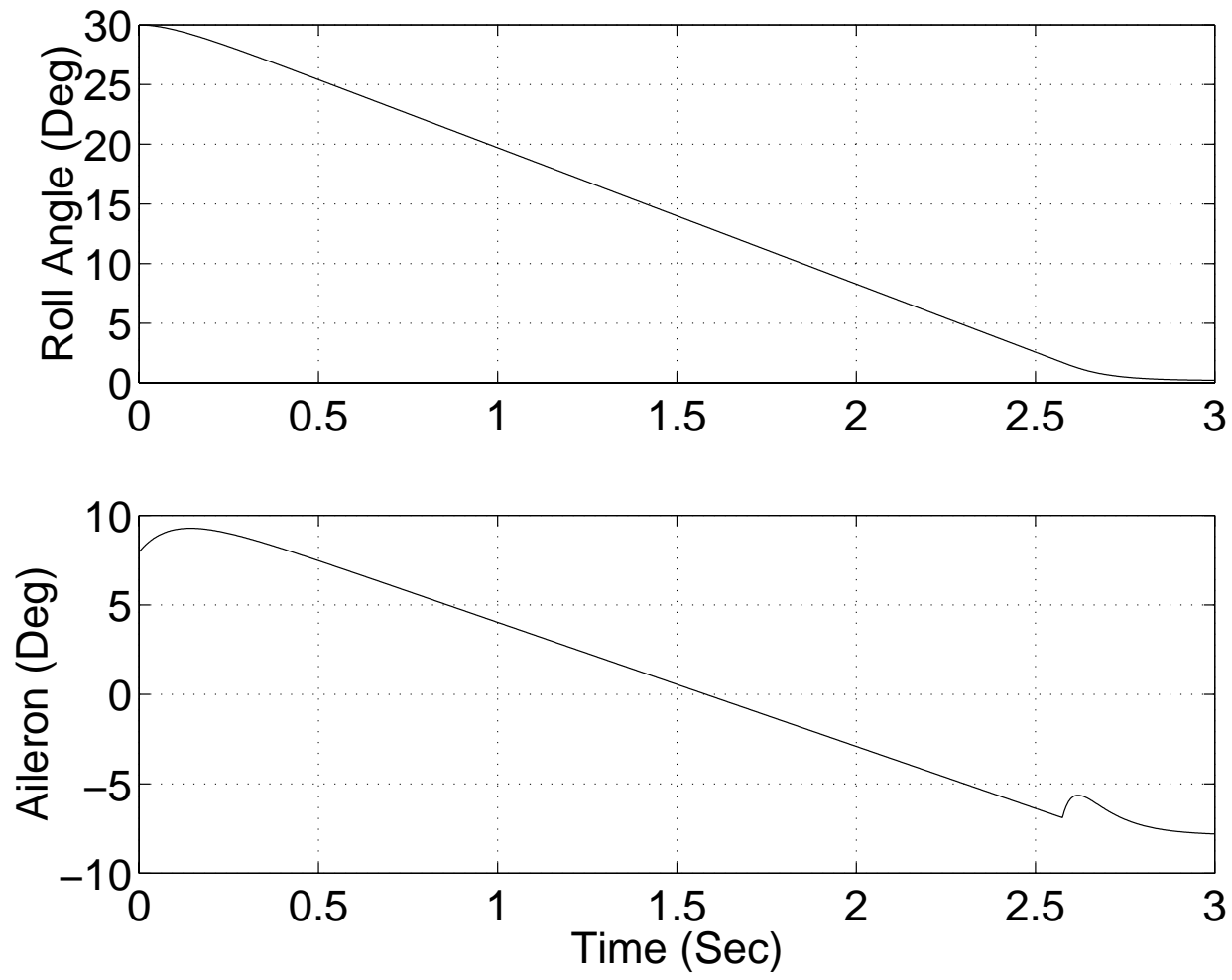


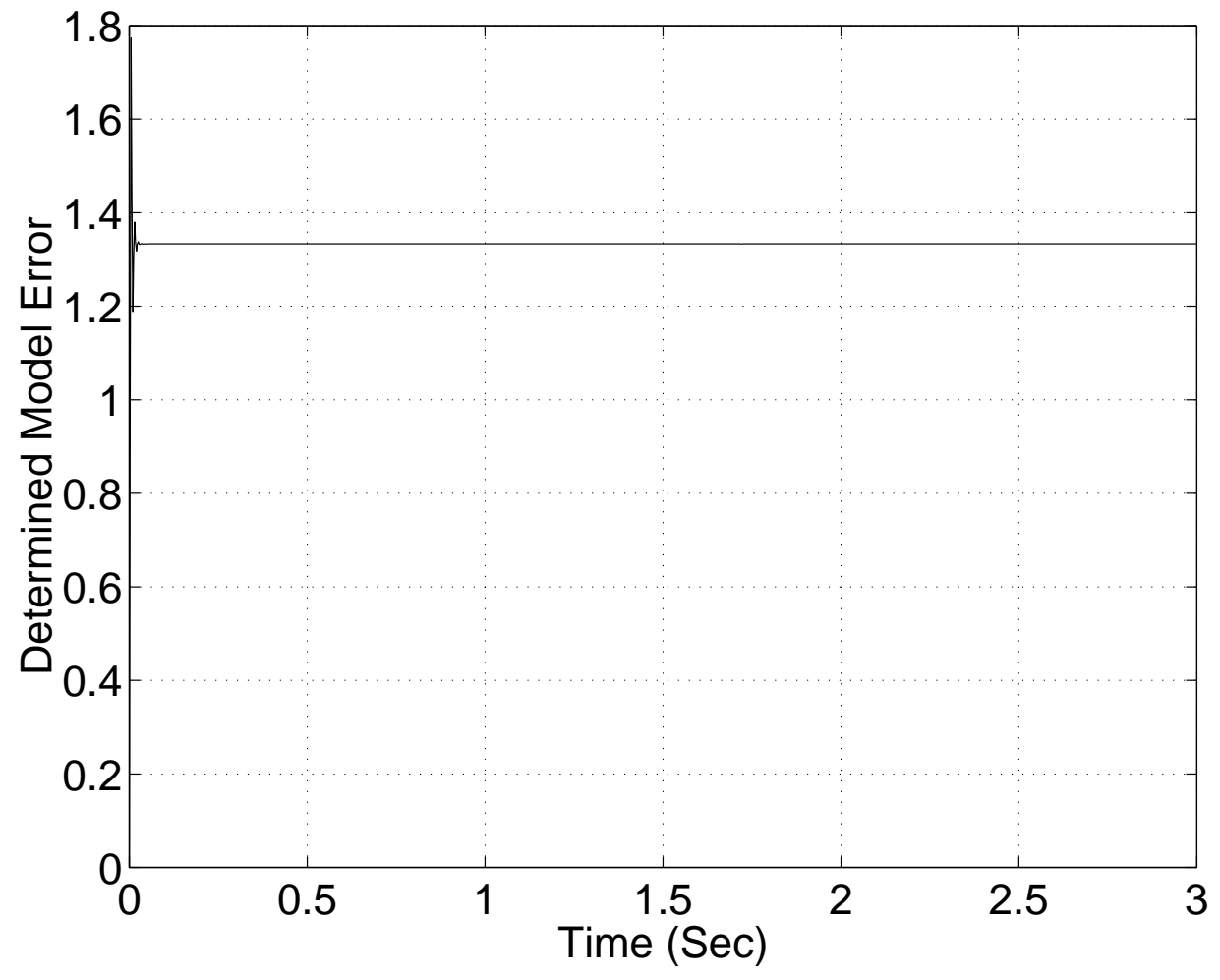



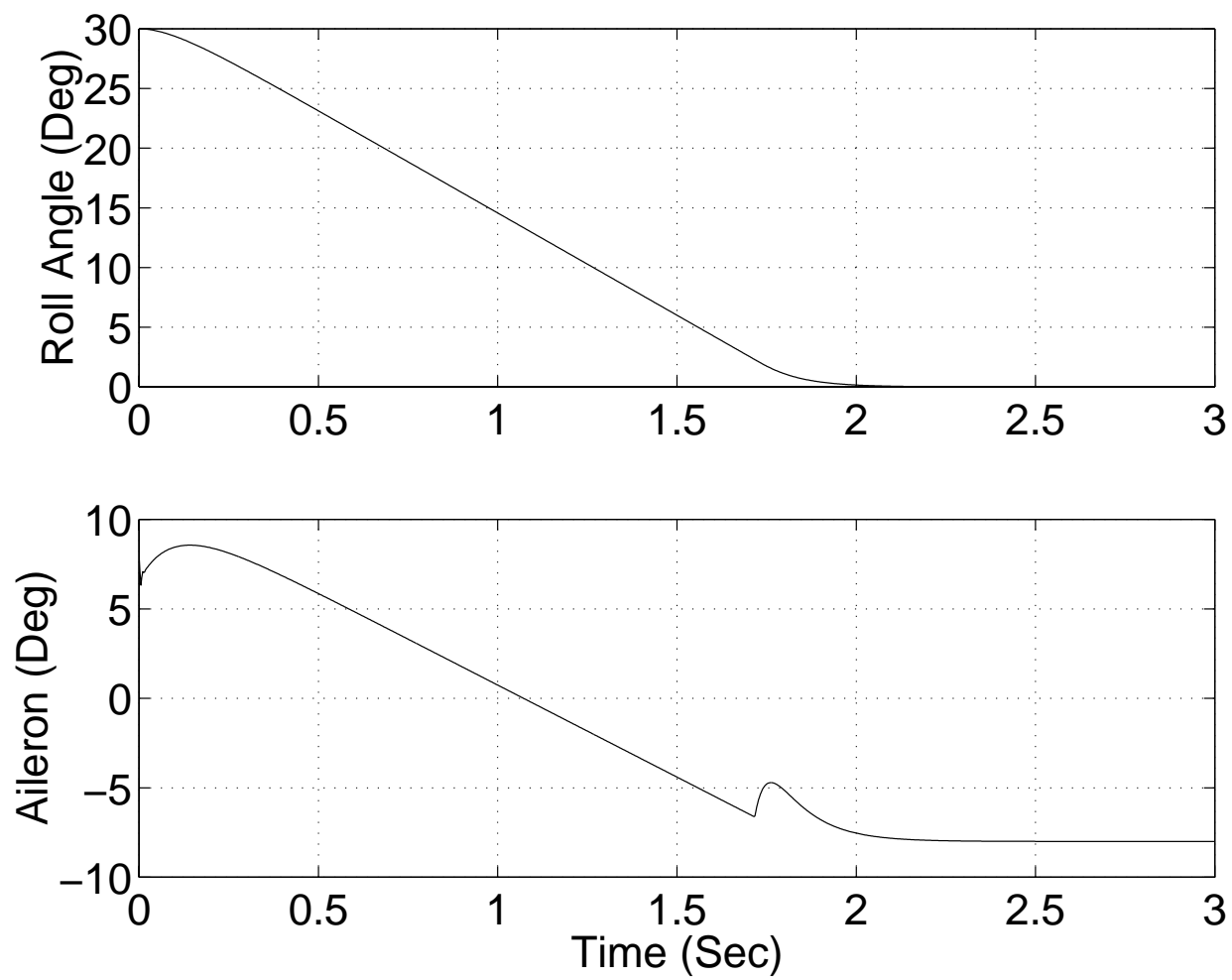

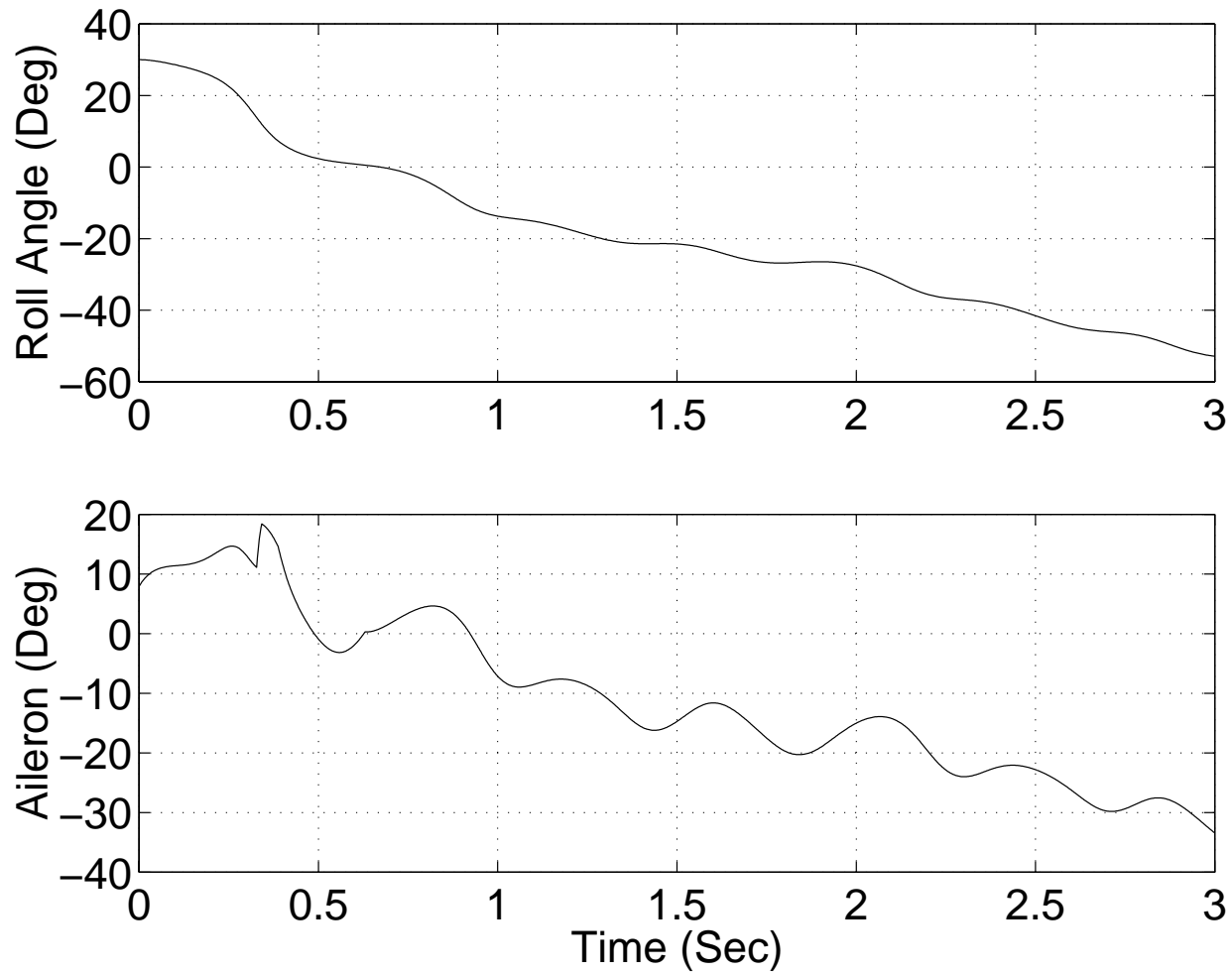


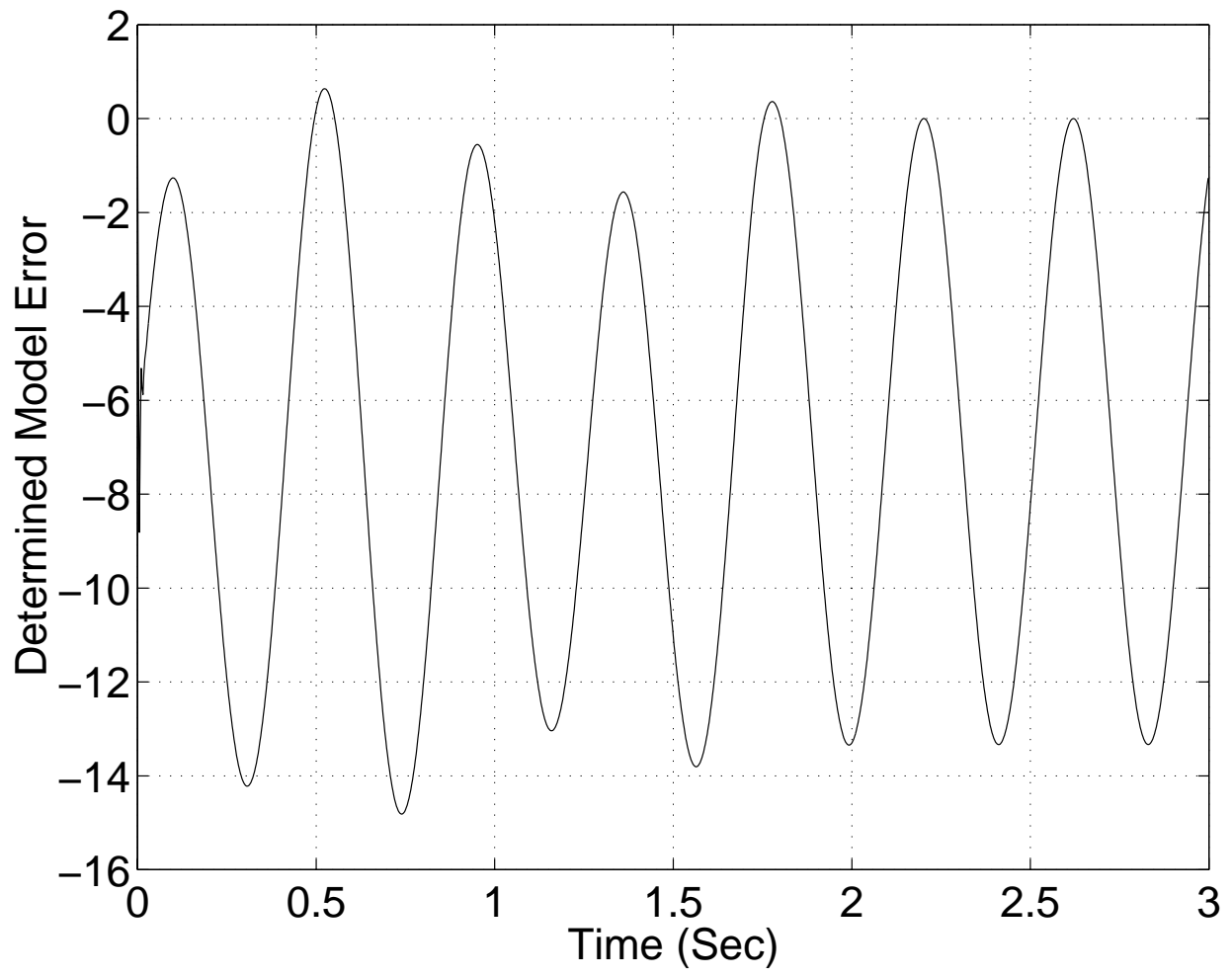



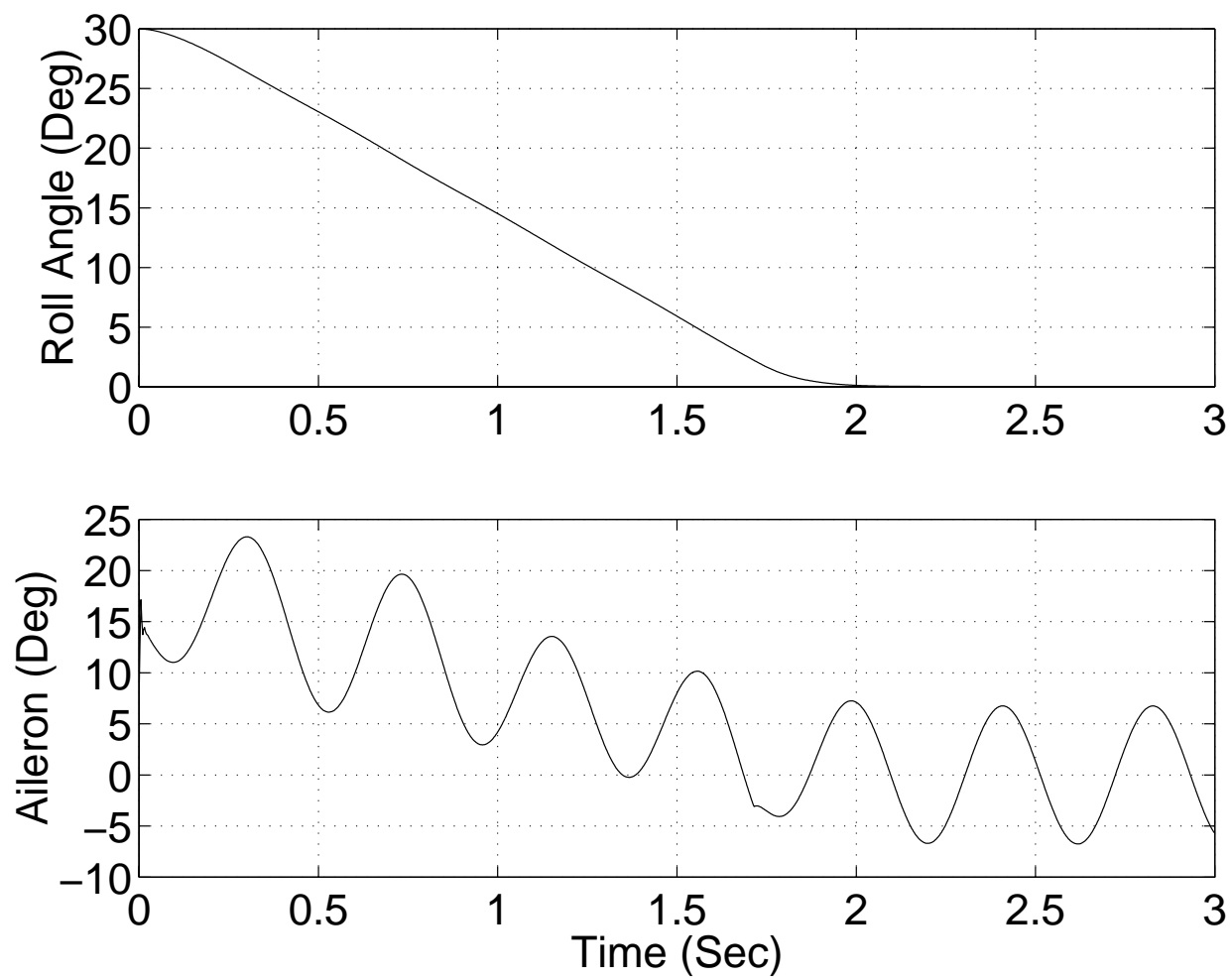

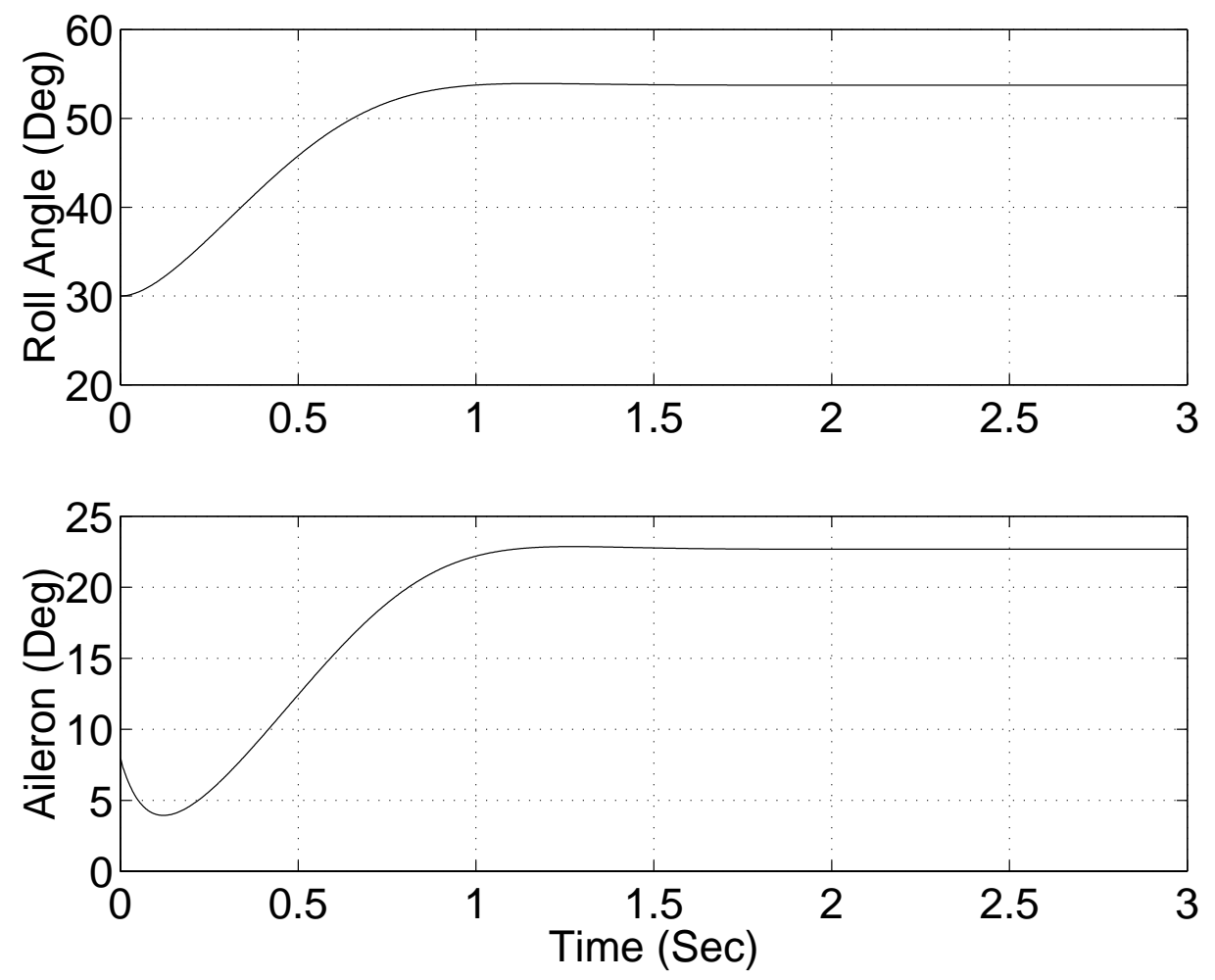


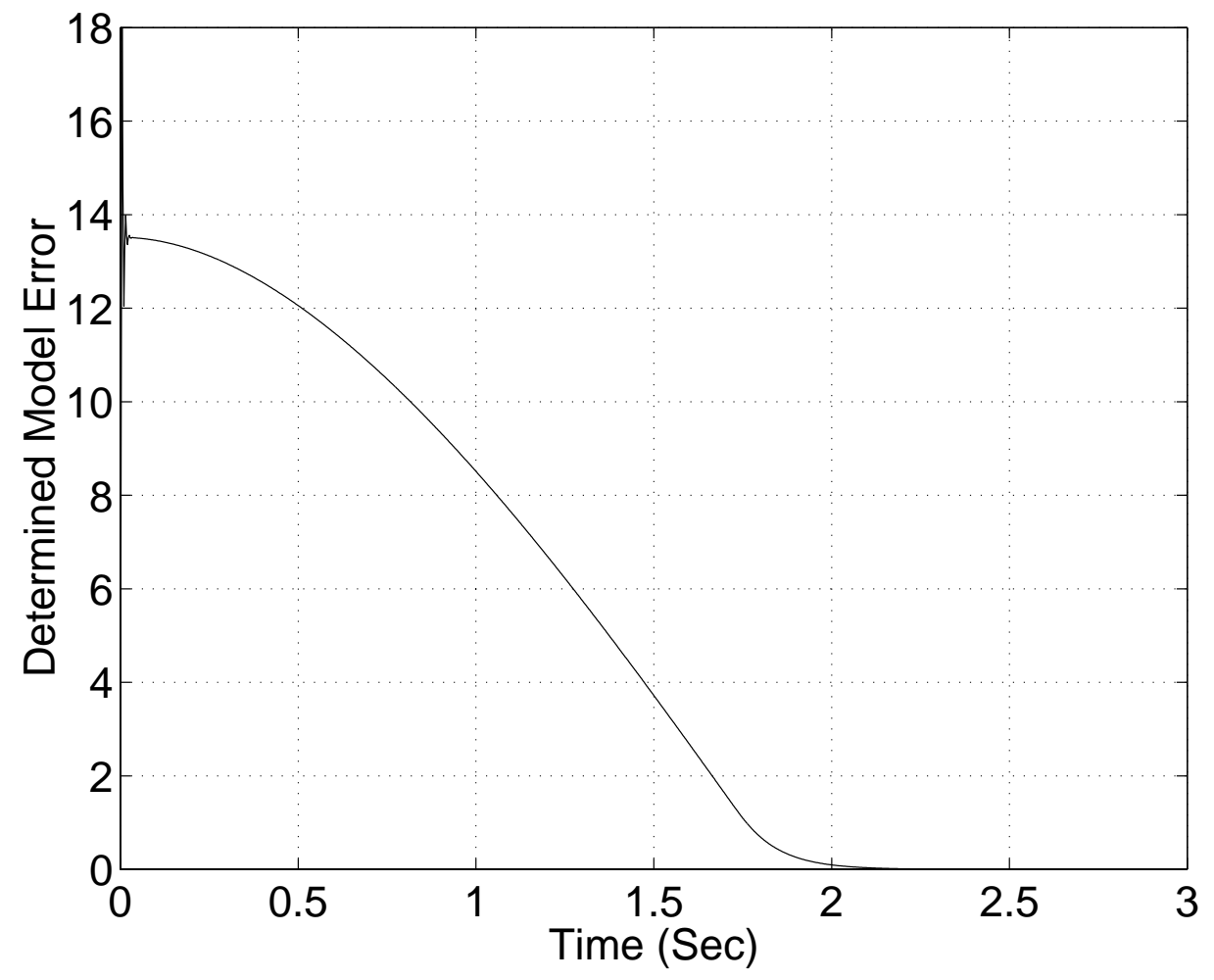



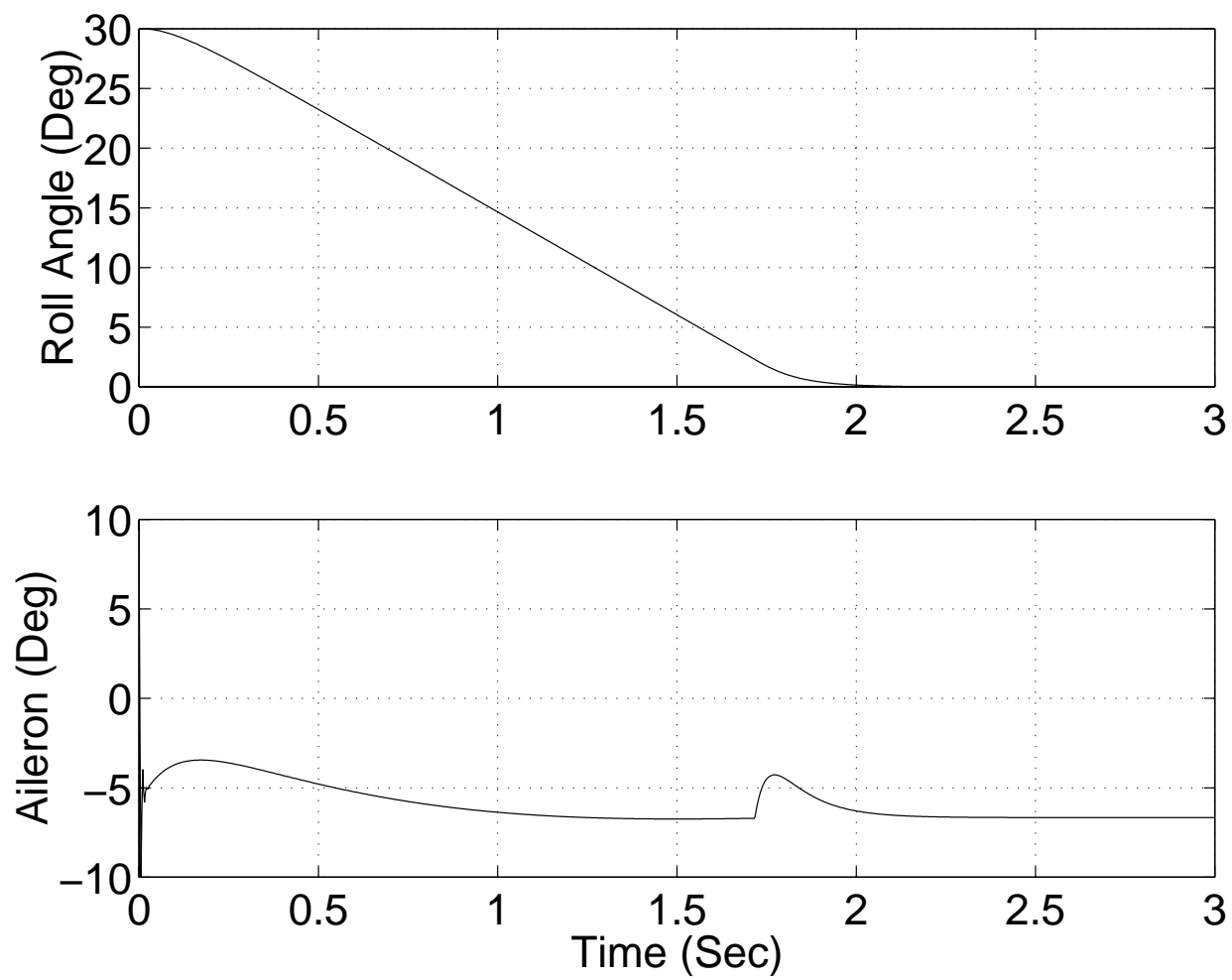